\title{
Fuzzy Design for Ship Heading Control Using ACFB for e-Navigation
}

Yancai Hu, Gyei-Kark Park, Taeho Hong, and Geonung Kim

Faculty of Maritime Transportation Sciences, Mokpo National Maritime University, Mokpo, Korea

\section{]jfis}

Received: Nov. 29, 2017

Revised : Dec. 17, 2017

Accepted: Dec. 20, 2017

Correspondence to: Gyei-Kark Park (gkpark@mmu.ac.kr)

(OThe Korean Institute of Intelligent Systems

(c) This is an Open Access article distributed under the terms of the Creative Commons Attribution Non-Commercial License (http://creativecommons.org/licenses/ by-nc/3.0// which permits unrestricted noncommercial use, distribution, and reproduction in any medium, provided the original work is properly cited.

\begin{abstract}
In this paper, a fuzzy robust design for ship heading control using ACFB (adaptive command filtered backstepping) is proposed considering the input saturation constraints and external disturbances for e-Navigation. This novel adaptive controller is constructed for the underactuated surface ship with rudder dynamics control system based on the Lyapunov stability theory, and this control algorithm guarantees the stability of the closed-loop system. T-S fuzzy logic system is employed to approximate nonlinear uncertainty in the control system. The proposed algorithm is developed by combining command filter and minimal learning parameter (MLP) techniques to compensate the control error. The substantial problems of "explosion of complexity" and "dimension curse" existed in the conventional adaptive backstepping technique are circumvented. It is convenient to implement for the e-Navigation application. Moreover, in order to deal with the effect of input saturation constraints, an auxiliary system is developed. Finally, the MATLAB simulation results of a case study of Ship YULONG are given to demonstrate the effectiveness and robustness of the proposed scheme.
\end{abstract}

Keywords: Ship heading control, T-S fuzzy logic system, Command filter, MLP, Input saturation, e-Navigation

\section{Introduction}

When facing the highly nonlinear ship dynamics and external disturbances of the environment, it is a great challenge for the ship to control to track the desired heading. Therefore a robust adaptive heading control is very important to the autopilot of e-Navigation. As we know, there is no doubt that the advanced ship-heading autopilot can enhance berth to berth navigation, and provide the e-Navigational solutions for the safety and efficiency of maritime transport.

In recent years, the ship heading control has always received considerable attention. Great efforts have been devoted to this prosperous topic and many significant results have been gained for the e-Navigation related ship autopilot design using the methods of backstepping, model reference and feedback linearization and so on. In [1], the backstepping and genetic algorithms were combined to design the adaptive heading controller. The paper [2] proposed an input-output linearization approach based on Lyapunov and Hurwitz for the nonlinear vessel steering system.

However, these exists a drawback of model-based methods that the explicit knowledge dynamics are necessary to be known. Recently, in order to overcome this drawback, in [3] the adaptive fuzzy logic control emerged to compensate the ship steering problems with uncertain ship dynamics. Due to the strong approximating ability, fuzzy logic system was used to 
approximate the unknown nonlinear uncertainties and promoted the development of ship motion control. Yang et al. [4] presented a novel robust adaptive fuzzy control algorithms based on small-gain and backstepping approach. Furthermore, in order to solve the "dimension curse" problem, MLP approach was utilized to reduce learning parameters and computation burden in paper [5], which is convenient to be implemented in applications.

The backstepping method has been a useful tool to design the controllers for the nonlinear systems since it was rst proposed. More recently, the adaptive backstepping control based on dynamic surface control (DSC) has been proposed in [6]. Nevertheless, these proposed controllers did not consider the errors arose from the filters. Fortunately, a novel command filtered method was provided in [7]. By the further study, the paper [8] researched the adaptive command filtered control.

Nevertheless, the input saturation is not considered in the above works. Hence, the design for the ships adaptive nonlinear systems have been studied in [9] considering the input saturation. The unknown dead-zone constraints were introduced based on command filter in [10]. However, the robust design of ships heading autopilot has not been considered by using command filter and MLP technique with rudder dynamic and external disturbances.

Motivated by the above observations, an adaptive fuzzy ship heading control based on command filter is proposed for nonlinear steering systems with input saturation. Fuzzy logic systems are employed to handle the nonlinear unknown terms in the steering system considering rudder dynamic.

\section{Problem Formulation and Preliminaries}

\subsection{Problem Formulation}

The nonlinear Norrbin ship mathematical model [1] is selected:

$$
T \dot{r}+r+\alpha r^{3}=K \delta
$$

where $T$ is the ship following index, $K$ is the ship turning ability index, $\delta$ is the rudder angle, $r$ is the angular deflecting rate, $\alpha$ is the coefficient of Norrbin, the rudder angle has limitation of $|\delta| \leq 35^{\circ}$.

The mathematical steering model [1] is added to the ship nonlinear Norrbin mathematical model, and it can be expressed as:

$$
T_{E} \dot{\delta}+\delta=K_{E} \delta_{E}
$$

where $\delta_{E}$ is the command rudder angle, $\delta$ is the actual rudder angle, $T_{E}$ is the time delay constant, and $K_{E}$ is the control gain.

Now we consider the nonlinear uncertain ship heading control system:

$$
\left\{\begin{array}{l}
\dot{x}_{1}=f_{1}+g_{1} x_{2}+\Delta_{1}, \\
\dot{x}_{2}=f_{2}(x)+g_{2} x_{3}+\Delta_{2}, \\
\dot{x}_{3}=f_{3}+g_{3} u+\Delta_{3}, \\
y=x_{1}
\end{array}\right.
$$

where $x_{1}=\psi, x_{2}=\dot{\psi}, x_{3}=\delta, u=\delta_{E}$, the external disturbances are simplified as $\Delta_{1}=\Delta_{2}=0, \Delta_{3}$ is unknown smooth functions. $f_{1}=0, g_{1}=1$. $f_{2}(x)$ is the unknown dynamics of control system, $g_{2}=K / T, f_{3}=-1 / T_{E}, g_{3}=K_{E} / T_{E}$.

\subsection{T-S Type Fuzzy Logic System}

Now, we briefly describe the structure of the T-S type fuzzy logic system. Generally, there are $\mathrm{N}$ rules in the fuzzy system, and each rule has the following form:

$$
\begin{aligned}
& R_{j}: \text { If } x_{1} \text { is } h_{1}^{j} \text {, AND } x_{2} \text { is } h_{2}^{j} \text {, AND } \cdots \text { AND } x_{n} \text { is } h_{n}^{j}, \\
& \text { then } y_{j} \text { is } a^{j} x \text { which is the function of } \\
& \qquad a_{1}^{i} x_{1}+a_{2}^{i} x_{2}+\cdots a_{n}^{i} x_{n} .
\end{aligned}
$$

$a_{i}^{j}, j=1,2, \cdots, N, i=1,2, \cdots, n$ are unknown constants, $h_{i}^{j}$ is the input variable, $a^{j} x$ is the output variable.

The product fuzzy inference is utilized to evaluate the ANDs of the fuzzy rules. After being defuzzified by a typical center average defuzzifier and an optimal parameter being defined, the output of the T-S fuzzy system is expressed as the following vector form:

$$
f(x)=\hat{f}\left(x, A_{x}\right)+\varepsilon=\xi(x) A_{x} x+\varepsilon,
$$

where $\xi(x)=\left[\xi_{1}(x), \xi_{2}(x) \cdots \xi_{N}(x)\right], \varepsilon$ denotes the approximating error.

The fuzzy basis function $\xi_{j}(x)$ and vector $A_{x}$ are as follows:

$$
\begin{aligned}
& \xi(x)=\frac{\prod_{i=1}^{n} \mu_{h_{i}^{j}}\left(x_{i}\right)}{\sum_{j=1}^{N} \prod_{i=1}^{n} \mu_{h_{i}^{j}}\left(x_{i}\right)}, \\
& A_{x}=\left[\begin{array}{llll}
a_{11} a_{12} & \cdots & a_{1 n} \\
a_{21} a_{22} & \cdots & a_{2 n} \\
\vdots & \vdots & \vdots & \vdots \\
a_{N 1} a_{N 2} & \cdots & a_{N n}
\end{array}\right] \text {, }
\end{aligned}
$$


where $\mu_{h_{i}^{j}}\left(x_{i}\right)$ is membership function.

We define five fuzzy sets for each variable with the labels of $A_{h_{1}^{j}}(N L), A_{h_{2}^{j}}(N M), A_{h_{3}^{j}}(Z E), A_{h_{4}^{j}}(P M)$ and $A_{h_{5}^{j}}(P L)$ which are characterized by the following five membership functions $\mu_{h_{1}^{j}}=\exp \left[-(x+1)^{2}\right], \mu_{h_{5}^{j}}=\exp \left[-(x-1)^{2}\right]$, $\mu_{h_{2}^{j}}=\exp \left[-(x+0.5)^{2}\right], \mu_{h_{4}^{j}}=\exp \left[-(x-0.5)^{2}\right], \mu_{h_{3}^{j}}=$ $\exp \left[-x^{2}\right]$.

\subsection{Other Useful Knowledge}

Assumption 1. The reference signal $x_{1 d}(t)$ is a sufficiently smooth function of $t$, and $x_{1 d}, \dot{x}_{1 d}, \ddot{x}_{1 d}$ are bounded.

Lemma 1. For any scalar variables $A$ and $B$, the following inequality holds

$$
|A B| \leq \frac{A^{2}}{4 \gamma^{2}}+\gamma^{2} B^{2},
$$

where $\gamma$ is a positive coefficient.

Lemma 2. Let $V:[0, \infty] \rightarrow R$ satisfies the inequality

$$
\dot{V} \leq-a_{0} V+b_{0}, t \geq 0,
$$

where $a_{0}$ and $b_{0}$ are two positive constants. Then

$$
V(t) \leq \frac{b_{0}}{a_{0}}+\left(V\left(t_{0}\right)-\frac{b_{0}}{a_{0}}\right) e^{-\left(t-t_{0}\right)}, \forall t \geq t_{0} \geq 0 .
$$

\section{Controller Design and Stability Proof}

In this section, there are 3 steps in backstepping design procedure. The virtual controller $\alpha_{2}, \alpha_{3}$ shall be developed at each step. Finally, considering input saturation of steering system, an overall control law is conducted at the last step.

Step1: Define the heading tracking error variable $z_{1}=x_{1}-$ $x_{1 d}$, one obtains

$$
\dot{\mathrm{z}}_{1}=x_{2}-\dot{x}_{1 d},
$$

where $x_{1 d}$ is the desire signal. Define the virtual control law $\alpha_{2}$ for $x_{2}$ in subsystem above.

Remark 1. In order to evade the repeated derivative of virtual control law and reduce the computing burden so-called as 'explosion problem' existing in conventional backstepping method, a designed filter $\dot{x}_{i c}=-\tau_{i}\left(x_{i c}-\alpha_{i}\right) i=2,3$ will be introduced in the following steps. Let $\alpha_{2}$ bypass the filter. The outputs $\alpha_{2}$ and $\dot{\alpha}_{2}$ of the filter are defined as $x_{2 c}$ and $\dot{x}_{2 c}$, respectively.
Now we define the compensated error variable as

$$
\bar{z}_{1}=z_{1}-s_{1}
$$

where the signal dynamic of $s_{1}$ is defined as

$$
\dot{s}_{1}=-k_{1} s_{1}+\left(x_{2 c}-\alpha_{2}\right)+s_{2} .
$$

Then the derivative of the compensated error can be converted into

$$
\dot{\bar{z}}_{1}=\bar{z}_{2}+k_{1} s_{1}+\alpha_{2}-\dot{x}_{1 d},
$$

where $\bar{z}_{2}$ is the second compensated tracking error variable defined as

$$
\bar{z}_{2}=z_{2}-s_{2},
$$

where the second tracking error variable is defined $z_{2}=x_{2}-$ $x_{2 c}$.

Now choose Lyapunov candidate as

$$
V_{1}(t)=\bar{z}_{1}^{2} / 2
$$

We choose the virtual control $\alpha_{2}$ as

$$
\alpha_{2}=-k_{1} z_{1}+\dot{x}_{1 d}
$$

Substituting the virtual control above into the derivative of (14), obtains

$$
\begin{aligned}
\dot{V}_{1}(t) & =\bar{z}_{1}\left(\bar{z}_{2}+k_{1} s_{1}-k_{1} z_{1}+\dot{x}_{1 d}-\dot{x}_{1 d}\right) \\
& =-k_{1} \bar{z}_{1}^{2}+\bar{z}_{1} \bar{z}_{2} .
\end{aligned}
$$

Step 2: Similarly, define the virtual control $\alpha_{3}$ for the subsystem above. According to (12) and (13), the signal dynamic of $s_{2}$ is defined as

$$
\dot{s}_{2}=-k_{2} s_{2}+g_{2}\left(x_{3 c}-\alpha_{3}\right)+g_{2} s_{3},
$$

where $x_{3 c}=x_{3}-z_{3}$ is obtained by letting $\alpha_{3}$ pass through the first-order filter.

Define the last tracking error variable as

$$
\bar{z}_{3}=z_{3}-s_{3}
$$

The unknown function $f_{2}\left(x_{2}\right)$ in (3) can be approximated by the T-S fuzzy logic system in (4). It is expressed as

$$
\begin{aligned}
f_{2}\left(x_{2}\right) & =\xi\left(x_{2}\right) A_{x} x_{2}+\varepsilon_{2} \\
& =\xi\left(x_{2}\right) A_{x} \bar{x}_{2}+\xi\left(x_{2}\right) A_{x} x_{2 c}
\end{aligned}
$$




$$
+\xi\left(x_{2}\right) A_{x} s_{2}+\varepsilon_{2}
$$

Then, one obtains

$$
\dot{\bar{z}}_{2}=\xi\left(x_{2}\right) A_{x} \bar{x}_{2}+\omega+g_{2} \bar{z}_{3}-\dot{x}_{2 c}+k_{2} s_{2}+g_{2} \alpha_{3},
$$

where $\omega$ is introduced for simplicity and can be expressed as

$$
\begin{aligned}
\omega= & \xi\left(x_{2}\right) A_{x} x_{2 c}+\xi\left(x_{2}\right) s_{2}+\varepsilon_{2}+\Delta_{2} \\
\leq & \left\|\xi\left(x_{2}\right)\right\|\left\|A_{x}\right\|\left\|x_{2 c}\right\|+\left\|\xi\left(x_{2}\right)\right\|\left\|A_{x}\right\|\left\|s_{2}\right\| \\
& +\left|\varepsilon_{2}\right|+\left|\Delta_{2}\right| \leq\left\|A_{x}\right\|\left\|\xi\left(x_{2}\right)\right\|\left\|x_{2 c}\right\| \\
& +\left\|A_{x}\right\|\left\|\xi\left(x_{2}\right)\right\|\left\|s_{2}\right\|+\left|\varepsilon_{2}\right|+\left|\Delta_{2}\right| \leq \lambda_{2} \varphi\left(x_{2}\right),
\end{aligned}
$$

where $\|\cdot\|$ denotes the Euclidean norm vectors.

Let $\lambda_{2}=\max \left(\left\|A_{x} x_{2 c}\right\|,\left\|A_{x} s_{2}\right\|,\left\|\varepsilon_{2}+\Delta_{2}\right\|\right), \varphi\left(x_{2}\right)=$ $1+\left\|\xi\left(x_{2}\right)\right\|$, and the normalized term $A_{x}^{m}=A_{x} / c_{2} \cdot c_{2}=$ $\left\|A_{x}\right\|$ is a unknown constant only for analytic purpose, $\left\|A_{x}^{m}\right\| \leq$ 1 and $\nu_{2}=A_{1}^{m} \bar{z}_{1}$.

Similarly, choose Lyapunov candidate as

$$
V_{2}(t)=V_{1}(t)+\bar{z}_{2}^{2} / 2+\Gamma^{-1} \tilde{\theta}_{2}^{\mathrm{T}} \tilde{\theta}_{2} / 2
$$

where $\Gamma$ is a design parameter.

Now design the virtual control law as

$$
\begin{aligned}
\alpha_{3}= & \frac{1}{g_{2}}\left[-k_{2} z_{2}-\bar{z}_{1}+\dot{x}_{2 c}-\frac{\hat{\theta}_{2}}{4 \gamma_{2}^{2}} \xi_{2}\left(\bar{x}_{2}\right) \xi_{2}^{T}\left(\bar{x}_{2}\right) \bar{z}_{2}\right. \\
& \left.-\hat{\theta}_{2} \varphi_{2}\left(\bar{x}_{2}\right) \tanh \left(\frac{\hat{\theta}_{2} \varphi_{2}\left(\bar{x}_{2}\right) \bar{z}_{2}}{\delta_{2}}\right)\right],
\end{aligned}
$$

where $\hat{\theta}_{2}$ are the estimates of $\theta_{2}, \tilde{\theta}_{2}=\theta_{2}-\hat{\theta}_{2}$.

The update control laws $\hat{\theta}_{2}$ will be designed as

$$
\begin{aligned}
\dot{\hat{\theta}}_{2}= & \Gamma_{2}\left[\bar{z}_{2}^{2} \xi_{2}\left(\bar{x}_{2}\right) \xi_{2}^{T}\left(\bar{x}_{2}\right) /\left(4 \gamma_{2}^{2}\right)+\varphi_{2}\left(\bar{x}_{2}\right)\left|\bar{z}_{2}\right|\right. \\
& \left.-\sigma_{2}\left(\hat{\theta}_{2}-\theta_{2}^{0}\right)\right] .
\end{aligned}
$$

Then, the time derivative of $V_{2}(t)$ is

$$
\begin{aligned}
\dot{V}_{2}(t)= & -k_{1} \bar{z}_{1}^{2}+\bar{z}_{1} \bar{z}_{2}+\bar{z}_{2} \dot{\bar{z}} z_{2}-\Gamma^{-1} \tilde{\theta}_{2} \dot{\theta}_{2} \\
= & -k_{1} \bar{z}_{1}^{2}-k_{2} \bar{z}_{2}^{2}+\bar{z}_{2}\left[c_{2} \xi\left(x_{2}\right) \nu_{2}+\omega_{2}+g_{2} \bar{z}_{3}\right] \\
& -\bar{z}_{2} \xi_{2}\left(\bar{x}_{2}\right) \xi_{2}^{T}\left(\bar{x}_{2}\right) z_{2} \hat{\theta}_{2} /\left(4 \gamma_{2}^{2}\right)+\sigma_{2} \tilde{\theta}_{2}\left(\hat{\theta}_{2}-\theta_{2}^{0}\right) \\
& -\bar{z}_{2} \hat{\theta}_{2} \phi_{2}\left(\bar{x}_{2}\right) \tanh \left(\hat{\theta}_{2} \phi_{2}\left(\bar{x}_{2}\right) \bar{z}_{2} / \delta_{2}\right) \\
& -\tilde{\theta}_{2} \bar{z}_{2}^{2} \xi_{2}\left(\bar{x}_{2}\right) \xi_{2}^{T}\left(\bar{x}_{2}\right) /\left(4 \gamma_{2}^{2}\right)-\tilde{\theta}_{2} \phi_{2}\left(\bar{x}_{2}\right)\left|\bar{z}_{2}\right| .
\end{aligned}
$$

It is worth to noting that $|A B| \leq A^{2} /\left(4 \gamma^{2}\right)+\gamma^{2} B^{2}$, then

$$
\bar{z}_{2}\left(c_{2} \xi \nu_{2}+\omega_{2}\right) \leq \theta_{2} \bar{z}_{2}^{2} \xi \xi^{T}\left(4 \gamma^{2}\right)+\theta_{2}\left|\bar{z}_{2}\right|\left|\varphi\left(x_{2}\right)\right|
$$

$$
+\gamma^{2} \nu_{2}^{T} \nu_{2}
$$

where $\gamma_{2}$ is a design parameter, $\theta_{2}=\max \left\{\lambda_{2}, c^{2}\right\}$.

According to the following inequality

$$
\tilde{\theta}_{2}\left(\hat{\theta}_{2}-\theta_{2}^{0}\right) \geq \tilde{\theta}_{2}^{2} / 2-\left(\theta^{*}-\theta_{2}^{0}\right)^{2} / 2 \text {. }
$$

Then

$$
\begin{aligned}
\dot{V}_{2}(t) \leq & -k_{1} \bar{z}_{1}^{2}-k_{2} \bar{z}_{2}^{2}+\gamma^{2} \nu_{2}^{T} \nu_{2}+\theta_{2}\left|\bar{z}_{2}\right|\left|\phi_{2}\left(\bar{x}_{2}\right)\right| \\
& +g_{2} \bar{z}_{2} \bar{z}_{3}-\hat{\theta}_{2} \phi_{2}\left(\bar{x}_{2}\right) \bar{z}_{2} \tanh \left(\hat{\theta}_{2} \phi_{2}\left(\bar{x}_{2}\right) \bar{z}_{2} / \delta_{2}\right) \\
& +\tilde{\theta} \phi_{2}\left(\bar{x}_{2}\right)\left|\bar{z}_{2}\right|-\sigma_{2} \tilde{\theta}_{2}^{2} / 2+\sigma_{2}\left(\theta-\theta_{2}^{0}\right)^{2} / 2 .
\end{aligned}
$$

Notice that

$$
\hat{\theta}_{2} \psi_{2}\left(\bar{x}_{2}\right)\left|\bar{z}_{2}\right|-\hat{\theta}_{2} \phi_{2} z_{2} \tanh \left(\hat{\theta}_{2} \phi_{2} \bar{z}_{2} / \delta_{2}\right) \leqslant \delta_{2} .
$$

Then, one has

$$
\begin{aligned}
\dot{V}_{2}(t) \leq & -k_{1} \bar{z}_{1}^{2}-k_{2} \bar{z}_{2}^{2}+g_{2} \bar{z}_{2} \bar{z}_{3}-\delta_{2} \\
& -\sigma_{2} \tilde{\theta}_{2}^{2} / 2+\sigma_{2}\left(\theta_{2}-\theta_{2}^{0}\right)^{2} / 2+\gamma^{2} \nu_{2}^{T} \nu_{2} .
\end{aligned}
$$

Step 3: The final control law shall be given in this step.

Consider the last tracking error variable $z_{3}$ and compensated tracking error variable $\bar{z}_{3}$, one has

$$
\dot{z}_{3}=f_{3}+g_{3} u-\dot{x}_{3 c} .
$$

The signal dynamic of $s_{3}$ in (17) is defined as

$$
\dot{s}_{2}=-k_{3} s_{3}
$$

Then, the derivation of $\bar{z}_{3}$ is as follows:

$$
\dot{\bar{z}}_{3}=f_{3}+g_{3} u-\dot{x}_{3 c}+k_{3} s_{3} .
$$

In order to reduce the input saturation effects in the system, an auxiliary design system is selected as follows:

$$
\dot{e}=-k e-f(\cdot) e / e^{2}+(u-v) \text { if }|e| \geq \varepsilon, \text { or } \dot{e}=0 \text {. }
$$

Then

$$
a \dot{z}_{3}=f_{3}+g_{3}(\Delta u+v)-\dot{x}_{3 c}+k_{3} s_{3},
$$

where $f(\cdot)=f\left(z_{3}, \Delta u\right), \Delta u=u-v, k>0, \varepsilon$ is a positive parameter, $e$ is a variable introduced to reduce the input saturation effects in the system. 
Choose the final control input as

$$
v=\left(-k_{3} z_{3}+\dot{x}_{3 c}-f_{3}+e-g_{2} \bar{z}_{2}\right) / g_{3} .
$$

Then the equation $(36)$ has

$$
\dot{z}_{3}=g_{3} \Delta u-k_{3} z_{3}+e+k_{3} s_{3} .
$$

Similarly, choose Lyapunov candidate as

$$
V_{3}(t)=V_{2}(t)+\bar{z}_{3}^{2} / 2+e^{2} / 2 .
$$

Then the time derivative of $V_{3}(t)$ is

$$
\begin{aligned}
\dot{V}_{3}(t)= & \dot{V}_{2}(t)+\bar{z}_{3} g_{3} \Delta u-k_{3} z_{3}^{2}+\bar{z}_{3} e-g_{2} \bar{z}_{2} \bar{z}_{3}+e \dot{e} \\
\leq & -k_{1} \bar{z}_{1}^{2}-k_{2} \bar{z}_{2}^{2}-k_{3} \bar{z}_{3}^{2}+\delta_{2}+\bar{z}_{3} e+\bar{z}_{3} g_{3} \Delta u \\
& +e \dot{e}+\gamma^{2} \nu_{2}^{T} \nu_{2}-\sigma_{2} \tilde{\theta}_{2}^{2} / 2+\sigma_{2}\left(\theta^{*}-\theta_{2}^{0}\right)^{2} / 2 .
\end{aligned}
$$

Choose the function of the auxiliary design as $f(\cdot)=f\left(\bar{z}_{3}, \Delta u\right)$ $=\left|g_{3} \bar{z}_{3} \cdot \Delta u\right|+\Delta u^{2} / 2$, notice that

$$
\begin{aligned}
& e \cdot \dot{e}=-k e^{2}-\left(\left|\mathrm{g}_{3} \bar{z}_{3} \cdot \Delta u\right|+\Delta u^{2} / 2\right) e^{2} / e^{2} \cdot+\Delta u \cdot e \\
& \begin{aligned}
& \Delta u \cdot e \leqslant \Delta u^{2} / 2+e^{2} / 2 \\
& \bar{z}_{3} e+\bar{z}_{3} g_{3} \Delta u+e \dot{e} \leq\left(\bar{z}_{3}^{2}+e^{2}\right) / 2+g_{3} \bar{z}_{3} \Delta u-k e^{2} \\
& \quad-\left|g_{3} \bar{z}_{3} \cdot \Delta u\right|-\left(\Delta u^{2}-\Delta u^{2}-e^{2}\right) / 2 \\
& \leq \bar{z}_{3}^{2} / 2-(k-1) e^{2} .
\end{aligned}
\end{aligned}
$$

Then substituting the inequalities (40), (41) into (39), one obtains

$$
\begin{aligned}
\dot{V}_{3}(t) \leq & -k_{1} \bar{z}_{1}^{2}-k_{2} \bar{z}_{2}^{2}-\left(k_{3}-1 / 2\right) \bar{z}_{3}^{2}+\delta_{2}-(k-1) e^{2} \\
& -\sigma_{2} \Gamma^{-1} \tilde{\theta}_{2}^{2} / 2 \Gamma^{-1}+\sigma_{2}\left(\theta-\theta_{2}^{0}\right)^{2} / 2+\gamma^{2} \nu_{2}^{T} \nu_{2} .
\end{aligned}
$$

Set $\sigma_{2} / 2 \Gamma^{-1}=\alpha_{0}$, and choose the parameters as $k=1+\alpha_{0}$, $k_{1}=k_{2}=\alpha_{0}, k_{3}=1 / 2+\alpha_{0}$, notice $\nu_{2}=A_{2}^{m} \bar{z}_{2}^{T}, A_{i}^{m} \leq 1$, $i=1,2,3$, then $\left\|\nu_{2}\right\| \leq\left\|A_{2}\right\|\left\|z_{2}\right\| \leq\left\|z_{2}\right\|$, choose $\gamma \geq 1$, one finally has

$$
\dot{V} \leq-2 \alpha_{0} V+\rho \leq c_{1} V+\rho,
$$

where $c_{1}=2 \alpha_{0}, \rho=\sigma_{2}\left(\theta-\theta_{2}^{0}\right)^{2} / 2+\delta_{2}$. Then one obtains

$$
V(t) \leq \rho / c_{1}+\left(V\left(t_{0}\right)-\rho / c_{1}\right) e^{-\left(t-t_{0}\right)}, \forall t \geq t_{0} \geq 0 .
$$

It can be seen that all the signals of the closed-loop system are uniformly ultimately bounded. For any $\mu_{1} \geq\left(\rho / c_{1}\right)^{1 / 2}$ and $T \geq 0$, there exists $\left\|\bar{z}_{1}(t) \leq \mu_{i}\right\|$ for all $t \geq t_{0}+T$. When the designed parameters are selected appropriately, $\rho / c_{1}$ can be arbitrarily small, and the tracking error can be made as small as possible. Thus the course controller design and stability proof are finished.

\section{Simulation}

In this section, simulation results are given on the base of an ocean-going training ship YULONG. The parameters are shown as below:

The length between perpendiculars is $L=126 \mathrm{~m}$, the moulded breadth is $B=20.8 \mathrm{~m}$, the designed load draft is $T d=8.0 \mathrm{~m}$, the square coefficient is $C b=0.681$, and the speed is $U=7.7$ $\mathrm{m} / \mathrm{s}$. The parameters of the ship nonlinear mathematical model can be calculated as $K=0.478, T=216, \alpha=30$. The initial states are zero. The desired reference signal is $\mathrm{x}_{1 d}=60^{\circ}$. The rudder angle has a limitation of $|\delta|_{\max } \leq 35^{\circ}$.

Choose the design parameters as $k=1.02, k_{1}=0.05$, $k_{2}=20, k_{3}=2.5, \tau_{2}=\tau_{3}=2.5, \Gamma_{i 2}=2, \sigma_{2}=0.05, \gamma_{2}=3$, $\hat{\theta}_{2}^{0}=0$, the initial value $e=0.1$. The external disturbance signals are chosen as $\Delta_{2}=0.0001 * \sin (0.1 * t)$.

Simulation results are shown as as follows:

In the simulation, the curve (a) in Figure 1 and the curve (a) in Figure 2 are the responses of ships course-keeping process of command filter based method and DSC based method respectively. It can be seen that the tracking error is almost zero at the time of $100 \mathrm{~s}$, and command filter based method performs a smaller error; the curves (b) in Figures 1 and 2 are the heading rates of the ship for command filter and DSC. The curves (c) and (d) in Figure 3 are the command and actual rudder angles of ship. The input saturation based autopilot is restrained by the maximum rudder angle. We can see from the course-keeping curve that the controller has fast response speed and performs well; Figure 4 shows the responses of the virtual control variables and command filter outputs in curves (e), (f). Obviously, all signals are reasonable and satisfactory for keeping a desired course using the proposed scheme in this paper.

\section{Conclusion}

This paper has discussed the problem of heading control for an underactuated surface vessel with rudder actuator dynamics and input saturation. This control algorithm is combined with command filtering technology and MLP approach, which 

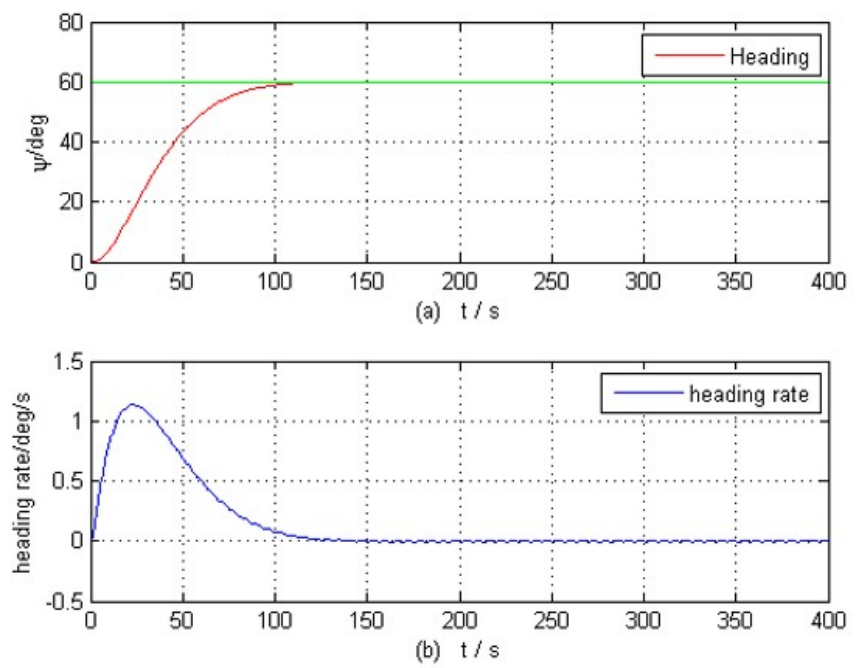

Figure 1. Time response of heading tracking error (a) and heading rate (b).

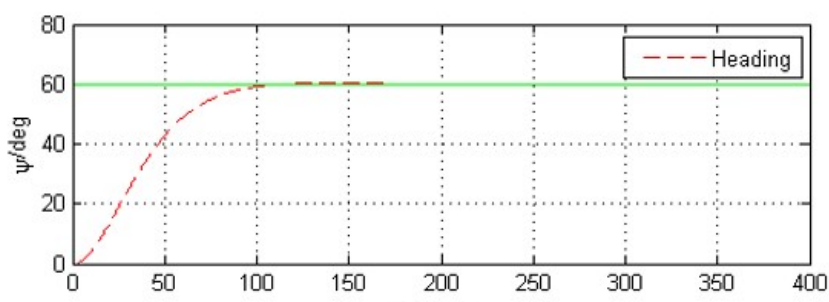

(a) $t / s$

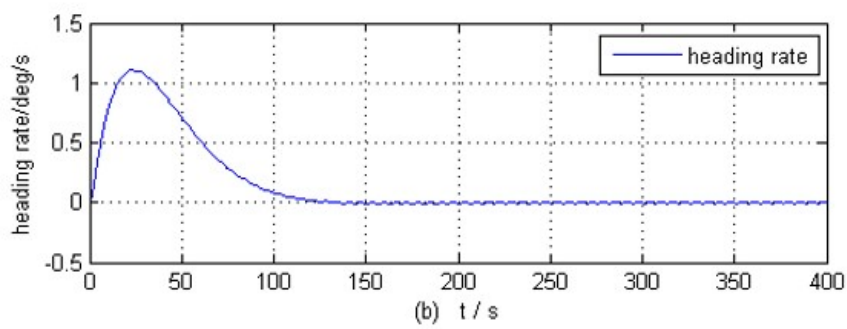

Figure 2. Time response of course tracking error (a) and heading rate (b) for DSC .

greatly reduces the complexity of the controller. The fuzzy logic system has been used to approximate the unknown nonlinear functions. This proposed scheme guarantees the stability of the closed-loop system and has a good performance. The simulation results validate the effectiveness and robustness of this proposed scheme. This proposed scheme will be available for implementing VMS (Vessel Monitoring System) for e-Navigation, contribute to the optimal route planning, and strengthen maritime safety.
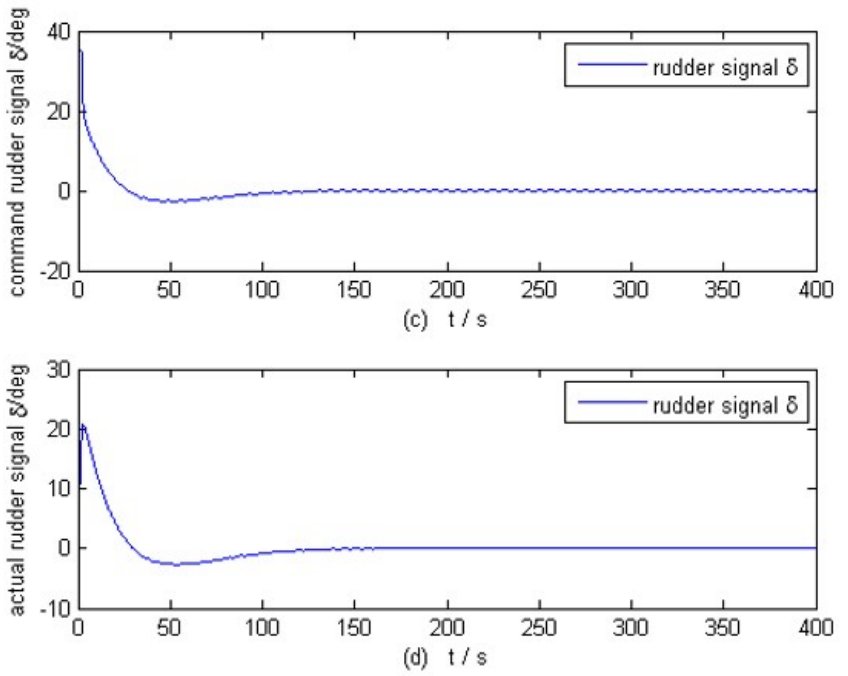

Figure 3. Time responses of command and actual rudder angles.
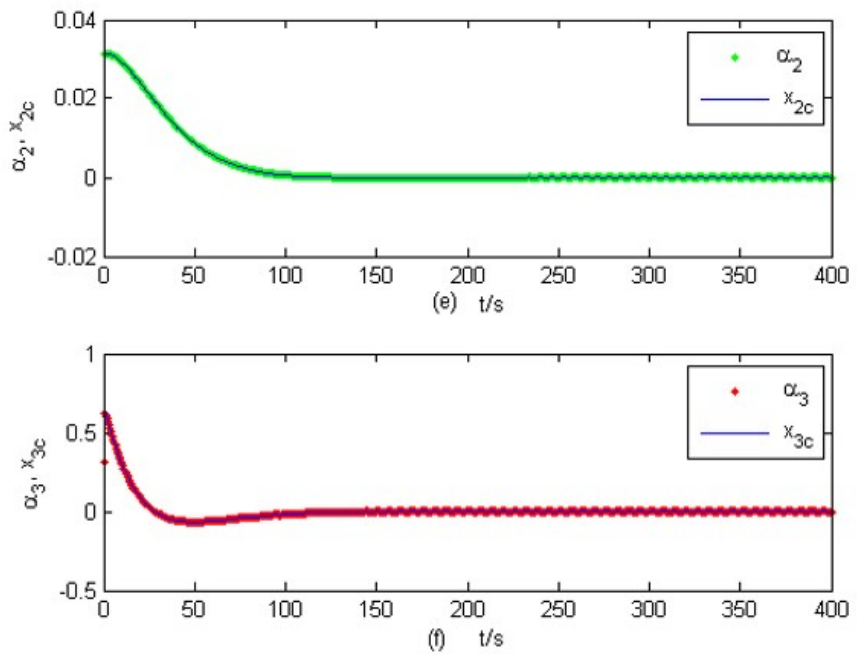

Figure 4. Time response of virtual control variables and the outputs of the command filters.

\section{Acknowledgement}

This research is a part of the project titled "SMART-Navigation project," funded by the Ministry of Oceans and Fisheries.

\section{Conflict of Interest}

No potential conflict of interest relevant to this article was reported. 


\section{References}

[1] A. Witkowska, M. Tomera, and R. Smierzchalski, "A backstepping approach to ship heading control," International Journal of Applied Mathematics and Computer Science, vol. 17, no. 1, pp. 73-85, 2007. http://doi.org/10.2478/ v10006-007-0007-2

[2] L. P. Perera and C. G. Soares, "Lyapunov and Hurwitz based controls for input-output linearization applied to nonlinear vessel steering," Ocean Engineering, vol. 66, pp. 58-68, 2013. https://doi.org/10.1016/j.oceaneng.2013. 04.002

[3] C. Liu, T. S. Li, and N. X. Chen, "Adaptive fuzzy control design of ship's autopilot with rudder dynamics," ICIC Express Letters, vol. 5, no. 3, pp. 767-773, 2011.

[4] Y. Yang, G. Feng, and J. Ren, "A combined backstepping and small-gain approach to robust adaptive fuzzy control for strict-feedback nonlinear systems," IEEE Transactions on Systems, Man, and Cybernetics-Part A: Systems and Humans, vol. 34, no. 3, pp. 406-420, 2004. http://doi.org/ 10.1109/TSMCA.2004.824870

[5] T. Li, R. Li, and J. Li, "Decentralized adaptive neural control of nonlinear interconnected large-scale systems with unknown time delays and input saturation," Neurocomputting, vol. 4, no. 14, pp. 2277-2283, 2011. https://doi.org/10.1016/j.neucom.2011.03.005

[6] T. S. Li, D. Wang, and W. Li, "A novel adaptive NN control for a class of strict feedback nonlinear systems," in Proceedings of American Control Conference, St. Louis, MO, 2009, pp. 2746-2951. http://doi.org/10.1109/ACC. 2009.5159999

[7] J. A. Farrell, M. Polycarpou, M. Sharma, and W. Dong, "Command filtered backstepping," IEEE Transactions on Automatic Control, vol. 54, no. 6, pp. 1391-1395, 2009. http://doi.org/10.1109/TAC.2009.2015562

[8] W. J. Dong, J. A. Farrell, M. M. Polycarpou, V. Djapic, and M. Sharma, "Command filtered adaptive backstepping," IEEE Transactions on Control Systems Technology, vol. 20, no. 3, pp. 566-580, 2012. http://doi.org/10.1109/TCST. 2011.2121907
[9] M. Chen, S. S. Ge, and Y. Choo, "Neural network tracking control of ocean surface vessels with input saturation," in Proceedings of the 2009 IEEE International Conference on Automation and Logistics, Shenyang, China, 2009, pp. 85-89. http://doi.org/10.1109/ICAL.2009.5262972

[10] J. Yu, P. Shi, W. Dong, and C. Lin, "Adaptive fuzzy control of nonlinear systems with unknown dead zones based on command filtering," IEEE Transactions on Fuzzy Systems, 2016. http://doi.org/10.1109/TFUZZ.2016.2634162

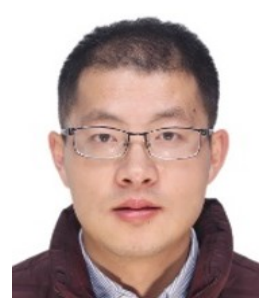

Yancai Hu is a Doctor candidate in Mokpo National Maritime University. His research interest includes maritime transport system.

E-mail: yancaihu@126.com

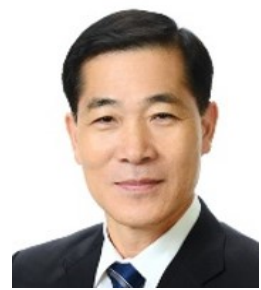

Gyei-Kark Park received the Ph.D. degree from Tokyo Institute of Technology. $\mathrm{He}$ is now a professor in Mokpo National Maritime University. His research interest includes fuzzy, game theory and international logistic.

E-mail: gkpark@mmu.ac.kr

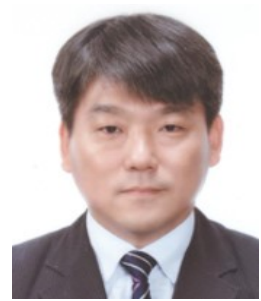

Geonung Kim received the Ph.D. degree from Korea University. He is now a prodessor in Mokpo National Maritime University. His research interest includes netword architecture and communication protocols. E-mail: kgu@mmu.ac.kr

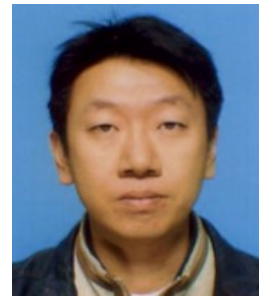

Taeho Hong received the Ph.D. degree from Kyushu University. He is now a assistant prodessor in Mokpo National Maritime Unversity His research interest includes information science and electrical engineering.

E-mail: ds1pnp@mmu.ac.kr 